\title{
Part 10: Digital Health
}

\author{
Telehealth Product and Service Design for an Age- \\ ing Population \\ Malcolm Fisk* \\ Centre for Computing and Social Responsibility, De \\ Montfort University, Gateway House, The Gateway, \\ Leicester, UK
}

Background: This paper draws on research undertaken within the European Commission funded PROGRESSIVE project (www.progressivestandards.org) on 'Standards around ICT and Active and Healthy Ageing'. The project ran from 2016 to 2019. It raised awareness of design imperatives relating to products and services that are useful when accessed by or provided for older people. The focus in this paper is on telehealth i.e. 'the means by which technologies and related services concerned with health and well-being are accessed by people or provided for them at a distance'. Within telehealth's remit are services and technologies from social alarms and telecare to the Internet of Things (IoT), smart homes and wearables.

The paper signals some shortcomings in telehealth product and service designs that can be a consequence of patronising or ageist beliefs about older people; or arise through overly commercial orientations. Some commercial orientations may be associated with a view of older people as a 'market' to be exploited rather than as contributors to economic and social life (as workers, carers, entrepreneurs, innovators and leaders).

Method: The paper explores ethical issues that should appropriately be taken into account for telehealth product and service design if the position, needs and choices of older people are to be properly understood. It undertakes a comparative analysis of the "ethical tenets' determined (following consultations with a 'task force' comprising seven older people convened by Age Platform Europe) in the PROGRESSIVE project, alongside other 'frameworks' and the methodologies that underpinned them such the PUX (Personal User Experience) guidelines (deriving from the work of the European Innovation Partnership on
Active and Healthy Ageing); the Age of No Retirement Intergenerational Design Principles; the ISO 26000 guidelines on 'Social Responsibility'; and the work of the European Commission programme for 'Responsible Research and Innovation'.

Key results: The key outcome of the comparative analysis is a succinct ethical framework for telehealth that has wider relevance for health and related social care services. The framework is considered in relation to a 'telehealth change imperative' that seeks to harness, in positive ways, the disruptive impact of new technologies; and to facilitate people's access to and adoption of such technologies (and use of services) in new ways. Conclusion: The paper argues that an appropriate ethical approach can underpin the role of telehealth in shaping future health services. It affirms that there is no 'doomsday' scenario of unsustainable services or a 'burden of care' because of the growing number of needy older people. Instead there is an opportunity for telehealth, subject to design considerations, relating to which

- through day to day technologies (smart phones, TV, wearables, etc.), older people are empowered by their access to information, employment and learning opportunities, social networks and more - including telehealth products and services;

- interoperable, accessible, usable, safe and otherwise well-designed telehealth products and services are available in ways that minimise intrusion and afford control by the older people who might wish to use them; and

- social norms are adopted that incentivise older (indeed, all) people to build their health literacy and engage in the management their health and related lifestyles.

Keywords: Telehealth, Standards, Ageing, Ethics, Design.

*Corresponding author. malcolm.fisk@dmu.ac.uk 


\author{
A Digital System Supporting Effective Remote \\ Multi-agency Home Visits \\ Fabio Ciravegna ${ }^{\mathrm{a}}$, Natalie Jones ${ }^{\mathrm{b}}$, Vitaveska Lanfran- \\ chi $^{\mathrm{a}}$, Jennifer Read ${ }^{\mathrm{c}}$, Emma Simpson ${ }^{\mathrm{b}}$, Claire Revitt ${ }^{\mathrm{e}}$, \\ Colette Fegan ${ }^{\mathrm{d}}$, Sarah Storey ${ }^{\mathrm{g}}$, Amber Wild ${ }^{\mathrm{f}}$ and Peter \\ Cudd $^{\mathrm{c}}$ \\ ${ }^{a}$ Department of Computer Science, University of \\ Sheffield, Sheffield, UK \\ ${ }^{\mathrm{b}}$ Sheffield Teaching Hospital NHS Foundation Trust, \\ Sheffield, UK \\ ${ }^{\mathrm{c}}$ School of Health and Related Research, University of \\ Sheffield, Sheffield, UK \\ ${ }^{\mathrm{d}}$ Department Allied Health Professions, Sheffield Hal- \\ lam University, Sheffield, UK \\ ${ }^{\mathrm{e}}$ Stroke Pathway Assessment and Rehabilitation Cen- \\ tre, Sheffield, UK \\ ${ }^{\mathrm{f}}$ Sheffield Health and Social Care NHS Foundation \\ Trust, Sheffield, UK \\ ${ }^{\mathrm{g}}$ Sheffield City Council, Sheffield, UK
}

Background: Unnecessary delayed discharge is a rising systemic problem. The total of delayed days in the UK has increased by 52\% between 2014 and 2017, the majority being related to patients with chronic or life-changing conditions awaiting for a care package to be organised at home. Sheffield Teaching Hospital focuses on the "discharge to assess" paradigm, where patients are transferred to a community setting and then assessed. This requires mechanisms to coordinate the care between city council, hospital, social care etc. that incorporate services that select, install and support assistive technology.

Method: We created a telepresence prototype to securely perform remote home assessments, sharing of notes, recording/taking photos across services. At AAATE 2018 we presented the initial prototype. In this paper, we describe the final prototype developed following a series of stakeholders focus groups and PPIs. The process requires the patient, a registered volunteer or a relative based in the patient's home to connect via a smartphone to the Hospital. Video connection is provided via the browser, no app installation or subscription needed. The patients will receive a link to a webpage via text message or email. Clicking on the link will open a direct video communication with the service provider. During the visit, the operator is in full control of the remote phone and camera, being able to turn on/off the flash (torch mode), flip the camera, take screenshots and videos, zooming in and out to appreciate details, etc. All data (notes, pictures, etc.) are stored securely on a central server.
The system also supports physical home visits by enabling taking pictures and writing notes that are then sent to the central server, therefore becoming a one stop shop for all discharge information for both physi$\mathrm{cal}$ and remote visits. As the visit may involve different services beyond the hospital (e.g. care homes, social services, city councils, etc.), the technology supports simultaneous participation of multiple users from multiple locations. That can include remote family members. This enables:

1) sharing of essential care planning and information among services;

2) user engagement and personalisation of care, through the ability of involving patients and remote family/carers in decision making;

3) an easy channel for post-discharge follow-up.

Key results: We contribute to the "discharge to assess" paradigm by enabling effective remote visits, information sharing and continuity of care. We enable patients and carers to fully participate in a delicate phase of the discharge process.

Conclusion: We present a system for remote visits supporting effective remote multi-agency home visits, streamlining the service, making patient and family involvement a corner stone of the discharge process hence improving quality of care and outcome of the process.

Keywords: discharge to assess, remote monitoring, remote visits.

${ }^{*}$ Corresponding author. E-mail: f.ciravegna@ shef.ac. uk

ProACT: Person-centred Digital Integrated Care for Adults Aged 65 Years and Over, Living with Multimorbidity

John Dinsmore ${ }^{\mathrm{a}}$, Emma Murphy ${ }^{\mathrm{a}}$, Mary Galvin ${ }^{\mathrm{a}, *}$, James Sheerin ${ }^{\mathrm{b}}$, Lorraine Tompkins ${ }^{\mathrm{b}}$, Evert-Jan Hoogerwerf $^{\mathrm{b}, *}$, An Jacobs ${ }^{\mathrm{c}}$, Cora Van Leeuwen ${ }^{\mathrm{c}}$, Myriam Sillevis Smitt ${ }^{\mathrm{c}}$, Valentina Fiordelmondo ${ }^{\mathrm{b}}$, Lorenzo Desideri $^{\mathrm{b}}$, Patricia Sheridan ${ }^{\mathrm{d}}$, Karen Coan ${ }^{\mathrm{d}}$, Suzanne Smith ${ }^{\mathrm{d}}$ and Julie Doyle ${ }^{\mathrm{d}}$

${ }^{\mathrm{a}}$ Trinity Centre for Practice and Healthcare Innovation, Trinity College Dublin, School of Nursing and Midwifery, D'Olier Street, Dublin 2, Ireland

${ }^{\mathrm{b}}$ AIAS Bologna onlus, Piazza della Pace 4/a, 40132 Bologna, Italy

${ }^{\mathrm{C}}$ imec-SMIT, Pleinlaan 9 second Floor, 1050 Brussels, Belgium

${ }^{\mathrm{d}}$ NetwellCASALA, Dundalk Institute of Technology, Co. Louth, Ireland 
Background: At present in Europe there are 50 million people living at any one time with multimorbidity. However, our healthcare systems have not been designed to effectively support these people in their daily care needs. ProACT (Integrated Technology Systems for ProACTive Patient Centred Care) is a digital health research programme funded under the European Union Horizon 2020 framework that seeks to address this problem by developing and evaluating a digital integrated care system to support older adults (65 years and over) living with multimorbidity. This presentation will outline the main findings of the ProACT project where the daily experiences of participants living with multimorbidity were captured and responded to through the design of digital health solutions.

Method: Vital to ProACT, was eliciting the voices of people living with multimorbidity as well as the various people caring for them (formal/informal caregivers, health care practitioners). A co-design methodology was adopted, making these stakeholders active participants in the design of ProACT. Across Ireland and Belgium the following was conducted over a 42 month project period; a qualitative user requirements study ( $n=124$ stakeholders); co-design workshops and usability testing ( $n=60$ stakeholders); a 12 month longitudinal, action research proof of concept trials (involving $n=120$ patients and their care networks). The primary trial sites were also supported by a transfer site in Italy consisting of 15 people with multimorbidity (PwM) and their care network, and a European transferability study was conducted to assess cultural, social, infrastructural, and political determinants for adoption and scalability of the system Traditional qualitative research techniques were combined with usercentred design methodologies to support the design, development and implementation of the ProACT digital system.

Key results: The main findings from the project will be presented, including the main themes that emerged from: the initial user requirements gathering (impact of multimorbidity; self-management; medication; knowledge, information and education; sources of support; communication; training; and technology); the codesign workshops and usability testing, which ensured that the ProACT technologies were designed to respond to previous themes; and finally results of the PoC trials, including themes pertaining to selfmanagement, the ProACT ecosystem, integrated care, and empowerment

Conclusion: Conclusions will demonstrate how digital health solutions can improve home-based inte- grated care, supporting older people with multimorbidity to live independently in their community. Furthermore, this project illustrates how using existing $\mathrm{Hu}$ man Computer Interaction (HCI) and behavioural science methodologies can improve the design, development and implementation of digital assistive technologies focused on older adult self-management of health and well-being within multi-stakeholder and diverse ecosystems of care and support.

Keywords: Digital Health, Behaviour Change, Multimorbidity, Digital Integrated Care, Ageing.

*Corresponding author. E-mail: galvinm6@tcd.ie

\section{Advancing Home-based Integrated care for Older adults with Multiple Chronic Conditions: Prelimi- nary Results from the Italian ProACT trial}

Arianna Gherardini ${ }^{\mathrm{a}, *}$, Lorenzo Desideri ${ }^{\mathrm{a}}$, Lisa Cesario $^{\mathrm{a}}$, Massimiliano Malavasi ${ }^{\mathrm{a}}$, Valentina Fiordelmondo $^{\mathrm{a}}$, Carlo Montanari ${ }^{\mathrm{a}}$ and Evert-Jan Hoogerwerf ${ }^{\mathrm{a}}$ a AIAS Bologna onlus, Piazza della Pace, Piazza della Pace 4/A, 40134 Bologna, Italy

Background: A key challenge for healthcare systems in Europe is to improve best practices around the provision of continued, well-coordinated, person-centred care for older adults with multimorbidity (OAM). At present, however, most health systems still focus on supporting a single disease framework of care, thus resulting in suboptimal outcomes for the health services users. ProACT is an EU-funded project aimed at developing and evaluating an ecosystem to integrate a wide variety of new and existing digital technologies to improve and advance home-based integrated care for older adults with multimorbidity, including associated co-morbidities. In detail, the ProACT kit integrates a wide variety of new and existing technologies to improve and advance home-based integrated care for older adults with multimorbidity with a view to (1) improve self-management of chronic conditions, (2) promote behavior change, and (3) increase integration between and within health \& social services. This study reports on the evaluation of the ProACT kit in the Italian context with a view to collect preliminary information on its usability and acceptability.

Method: The study followed a mixed quantitativequalitative research design. In this contribution we report on the qualitative data collected. A total of 10 users (age range 65-83 years) were asked to use the ProACT kit in their homes on a daily basis. The basic ProACT kit included the following devices: a smart- 
watch, a digital weight scale, and a digital blood pressure monitor. A tablet was given to the users to allow the visualization of the readings from the devices. Health professionals were also given the possibility to read each users' reading from a dedicated web interface. After 1 month of use, users participated in two focus groups to assess their views and experiences about the ProACT kit. A thematic analysis was conducted on the transcripts.

Key results: Three main themes were identified to explain how ProACT can facilitate self-management and behavior change as well as identifying the barriers to its adoption: Context, Process, and Outcome. The Context domain encompasses individual, family, and environmental factors. The process domain refers to procedural aspects of self-management, including specific behavior change strategies (goal setting, selfevaluation, and self-monitoring). The outcome domain includes short-term outcomes, such as successful symptom management and changes in health behaviors, which over time might result in distal outcomes, including reduced health system utilization and costs. To understand the potentials of ProACT to foster integrated care for people four dimensions of integration were further identified: Organizational, Functional, Service and Clinical.

Conclusion: The context in which this study was carried - as well as potential users of digital health technologies - seems to be ready to accept digital transformation of health and social services, providing that technologies are considered as a support to human activities and not merely substitutes. Technology should be designed taking into account diverse users' abilities and needs that can change over time. Context-related factors driving the organizational change needed to ease the adoption of digital health devices by all stakeholders need to be identified.

Keywords: Integrated care, ageing, digital health, behavior change

*Corresponding author. E-mail: agherardini@ausilio teca.org

Why do Dutch Older Adults Use Online Community Care Platforms, or Not?

Sarah Willard ${ }^{\mathrm{a}, \mathrm{b}, *}$, Marieke Spreeuwenberg ${ }^{\mathrm{a}, \mathrm{b}}$, Erik van Rossum $^{\mathrm{a}, \mathrm{b}}$, Nadine Spierts ${ }^{\mathrm{a}}$ and Luc de Witte ${ }^{\mathrm{a}, \mathrm{c}}$

${ }^{a}$ Centre of Innovative Care and Technology (EIZT),

Zuyd University of Applied Sciences, Henri Dunantstraat 2, 6419PB, Heerlen, The Netherlands

${ }^{\mathrm{b}}$ School CAPHRI, Care and Public Health Research
Institute (CAPHRI), Faculty of Health, Medicine and Life Sciences, Maastricht University, Maastricht, The Netherlands

${ }^{\mathrm{c}}$ Centre for Assistive Technology and Connected Healthcare (CATCH), University of Sheffield, Sheffield, UK

Background: The ageing of the Dutch population has led to healthcare reforms in which local governments encourage older adults to age-in-place and to self-manage their (health)care, if possible in collaboration with their social network and community. Prior research indicates that online communities can have a positive impact for older adults on civic participation, maintaining and developing social relationships and the exchange of various forms of social support. An example is an online community care platform (OCC-platform). Via OCC-platforms older adults can access applications that aim to support civic and social participation. The current study explores a Dutch OCC-platform implemented through an intensive usercentered process that took more than a year. Despite the efforts to implement this platform that fulfilled user requirements and conformed to important prerequisites distinguished by previous research, it was barely used by older adults. The purpose of this study was to gain insight in the experiences of older adults with the platform and their motives to adopt or reject it.

Method: In this qualitative study, three panels of older adults $(\mathrm{N}=16)$ were interviewed in focus groups: 1) digitally skilled platform users, recruited online via the OCC-platform, 2) digitally poor platform users, recruited via a course about digital skills, and 3) nonusers, who were members of a local activity-based group which was intensively involved in the implementation process but nonetheless chose not to use the platform. The Consolidated Framework for Implementation Research (CFIR) was used for the development of the interview guide and during the directed content analysis of the collected data.

Key results: The user-panels indicated that they barely used the platform and that it did not have any positive impact on their civic or social participation. Furthermore, all panels (users and non-users), predominantly gave motives for rejecting the OCC-platform, which was seen as: 1) externally developed, not accessible or user friendly and its information content as either outdated or as not matching expectations; 2) merely providing information one-sidedly and not as a twoway communication platform for facilitating civic and social participation; 3) intended for other people than themselves, i.e. "we are not old or frail". Finally, the 
non-users explained their lack of engagement in the implementation process due to interference and influence of other stakeholders; the (implementation of the) OCC-platform was not viewed as a self-made choice but rather as imposed on them by professionals and municipality-agents.

Conclusion: The OCC-platform was largely rejected whilst it conformed to the user(-interface) requirements. Users were intensively involved in its development. We expected the platform to be usable, accessible and functional. The results invalidate these expectations. This raises fundamental questions about how to co-create such platforms and how to make sure they are used. Working with end users in the development process doesn't guarantee that the product actually meets a felt need. Future recommendations are: 1) to perform a comprehensive holistic study on the inner setting of a given community and 2) to employ 'outof-the-box' methods to accomplish the latter, such as 'Co-creation' and 'Design Thinking'.

Keywords: ageing-in-place, online community platform, social network, civic participation, social support *Corresponding author. E-mail: sarah.willard@zuyd.nl

\section{Opportunities for Multimedia Tools to Connect Care Services}

Natalie Jones $^{\mathrm{a}, *}$, Peter Cudd ${ }^{\mathrm{b}}$, Claire Revitt ${ }^{\mathrm{a}}$, Emma Simpson $^{\mathrm{a}}$, Jennifer Read ${ }^{\mathrm{a}}$ and Colette Fegan ${ }^{\mathrm{C}}$

${ }^{a}$ Occupational Therapy Department, G Floor, Sheffield Teaching Hospitals NHS Foundation Trust, 10 Glossop Road, Sheffield S10 2JF, UK

${ }^{\mathrm{b}}$ Faculty of Medicine, Dentistry and Health, School of Health and Related Research, University of Sheffield, Sheffield, UK

${ }^{\mathrm{c}}$ Sheffield Hallam University, Sheffield, UK

Background: Making use of digital technologies to support delivering AT through a remote service is occurring somewhat haphazardly. The rehabilitation field is already advancing remote services and its application in AT services needs to catch up.

ATs can be useful tools for meeting support needs when people are discharged from acute care. In many nations multiple care providers, other agencies, family and friends form the network of stakeholders in determining needs and solutions. The who, the way and the what of the services involved vary across diseases and disability. The nett result is that real world services are complex and often service users cannot directly participate in decision making, and AT needs to be considered alongside other interventions. Previously work has explored digital multimedia tools facilitating communication and sharing in the network but from the perspective of acute service discharge to people's own homes. Now what the technology could empower within current services, involving the perspectives of the community services and agents, is being investigated.

Method used: A collaborative and co-production qualitative approach is being employed. All the main stakeholder service providers within one city (3) are part of the team and guiding the engagement of their staff $(\mathrm{N}=45-75)$ in focus group based workshops(3); in which, after introduction to the technology they can speculate and suggest how it might be useful in their routine practice. A synthesis from the workshop data will be reviewed by the project team and by service users. Indeed throughout service users have been and are being consulted to express their perspectives on the usefulness and acceptability of services/uses being suggested. All the stakeholder groups contacted so far have staff enthusiastic to take part - both in the collaboration and in the workshops. Three workshops have been held, 52 attended, this included members of actively supporting non-governmental organisations.

Key results: Whether service users (needing AT) have their needs assessed before discharge or at discharge from acute care having the direct involvement of the service users is highly desirable.

Preliminary analysis of the workshop data indicates that there are many potential novel uses and some suggestions for modifications to the technology have been identified; as expected there are some concerns about security and data governance. This approach is effective in triggering inventive and critical thinking for the use of AT in clinical practice. Early on the consultations outside the workshops have been important. For instance, the perspective of the social care service provider is very different and will significantly impact our final findings.

Conclusion: Embedding the research and development through involving stakeholders is empowering to answering real world challenges. However it also means that the challenges are more significant. Nonetheless, the positive attitudes and responses to involvement in the project are encouraging that multimedia tools will be acceptable to staff and there is an opportunity to improve intra- and inter- service operations and delivery of AT solutions.

Keywords: Multi-media tools, remote services, coproduction, digital, communication.

${ }^{*}$ Corresponding author. E-mail: Natalie.Jones@sth. nhs.uk 


\section{A Systems Approach to the implementation of a national model of Assistive Technology Service De- livery: Challenges and Opportunities Designing for the future: A Systemically Viable Assistive Technol- ogy Service for Disabled and Older People in Ire- land \\ Joan O’Donnell, Siobhán Long and Pierce Richardson Enable Ireland, Ireland}

Background: Irish Assistive Technology services have been under-resourced and inequitable by comparison with Australia, Finland and Denmark. This research sought to understand service delivery across education, health, employment and independent living, identify gaps, and establish the cost benefit of AT provision. The research sought to develop a systemically viable model for a coherent service, in a world characterized by Volatility, Unpredictability, Complexity and Ambiguity (VUCA), where public budgets are in flux, and technological advances are rapid, that understood the complexity of disability as a "wicked problem".

Method used: Soft Systems Methodology guided a systemic inquiry into current service levels: this included 15 stakeholder interviews and two group consultations using World Café methodology with 57 participants across state service providers, AT users, therapists, makers, academics and industry. Participants self-selected as members of a community of practice for AT. In accommodating the worldviews of different stakeholders, it became clear that a multifaceted approach was required that was flexible enough to respond to the complexity of disability and the diversity of people's circumstances and AT needs. It would need to encompass the whole journey from assessment to provision, training and follow up support. 3 case studies of individuals in different life situations were developed to establish a rationale for the cost benefit of an adequate AT service. They indicated the potential for significant savings if people are supported via AT to engage in education, work and live independently.

Key results: A qualitative analysis of current services indicated discrepancies in service provision and budgets nationally: there was a lack of clarity on how budgets were spent and the employment support budget was under-utilised. There was little evidence of cooperation across departments resulting, for example, in difficulties for people transitioning to take their AT from school to work. The research also indicated very different understandings of the relevance of AT: the Department of Education is forward looking in introducing AT at preschool level, yet in work support mechanisms are outmoded. Collaboration between different agencies is limited, with little political understanding of the potential of Assistive Technology.

Conclusion: The research confirmed that Ireland's AT services were sufficiently underdeveloped and siloed to require the design of a coherent national model. We proposed the development of an eco-system to create a comprehensive service to include a policy commitment from central government and a Central Co-ordinating Agency to take ownership of procurement, funding, co-ordination of service provision, training and information provision and create space for innovation in this domain. Included, is the concept of the AT Passport - a single resource to support Assistive Technology users to access the supports they need. The current political and policy environment points to an urgent need for the design of a central resource at a strategic government level. This is systemically desirable and is on the cusp of becoming an idea "in good currency". We also postulate that it has the potential to lead the way in redefining approaches to addressing other complexities of living with a disability in a climate-change world. ${ }^{*}$ Corresponding author. E-mail: slong@enableireland. ie 